\title{
Die blockierte Erinnerung
}

\section{Portugals koloniales Gedächtnis und das Ausbleiben kritischer Diskurse 1974-2010}

\author{
von Isabel dos Santos Lourenço und Alexander Keese*
}

\begin{abstract}
In 1974, the Portuguese colonial empire fell with the end of the authoritarian Estado Novo in the metropolis. The strong links between the success of the regime and the maintenance of the colonial territories, constantly insisted upon by leading figures in Portuguese politics over this period, rendered a post-revolutionary discussion about the nature of the colonial experience and the violent way towards decolonization, feasible. However, there was no such discussion, and the imperial past of the Estado Novo period remains outside any form of commemoration or critique. Several elements effectively blocked the colonial memory, including the colonial structures and personal continuities.
\end{abstract}

\section{1961/2011 - Ein stilles Jubiläum}

Der Beginn der antikolonialen Aufstände in Portugals afrikanischen Kolonien jährt sich 2011 zum fünfzigsten Mal und dies hat zumindest in der akademischen Welt einige praktische Effekte. Das Instituto de Ciências Sociais und das Centro de Estudos Africanos do Instituto Universitário de Lisboa, beide in Lissabon, bereiten für den Juni 2011 eine größere Konferenz zum Ende des portugiesischen Kolonialreiches vor. Das Centro de Estudos Africanos da Universidade do Porto stellt für das Frühjahr ein thematisches Dossier in Africana Studia, der einzigen regelmäßig erscheinenden Zeitschrift zu Afrikastudien in Portugal, über den Beginn der antikolonialen Kriege zusammen. In den etablierten universitären Institutionen ist fraglos das grundsätzliche Bewusstsein dafür aufgekommen, dass die Dekolonisation des portugiesischen Imperiums ein bedeutendes Thema in der Geschichte des Landes im 20. Jahrhundert darstellt. Es ist freilich sogleich anzumerken, wie Margarida Calafate Ribeiro bereits zutreffend betont hat, dass diesem grundsätzlichen Bewusstsein die praktische "Inexistenz" von historischen und sozialwissenschaftlichen Studien zum Dekolonisationsprozess gegenübersteht. Die für 2011 angedachten akademi-

* Die Autoren sind tätig am Centro de Estudos Africanos da Universidade do Porto (CEAUP). Alexander Keese dankt dem ERC Starting Grant innerhalb des Rahmenprogramms FP7 der Europäischen Union für die großzügige Unterstützung seiner Arbeit. 
schen Reaktionen entsprechen dann auch noch keineswegs der Dimension des kommentierten Ereignisses. ${ }^{1}$

In den Auseinandersetzungen, die man als "gesamtgesellschaftliche Diskussion“ bezeichnen könnte, spielt die Dekolonisationserfahrung eine noch weitaus geringere Rolle. ${ }^{2}$ Es existiert kein Mechanismus, mit welchem dieses Thema unter den politischen Eliten erinnert oder in breiteren sozialen Schichten diskutiert würde. Dies ist umso bemerkenswerter, als ein vergleichsweise hoher Prozentsatz der vor 1955 geborenen männlichen Portugiesen aktiv im Rahmen militärischer Operationen in den Kolonialgebieten präsent war. Damit waren auch die meisten portugiesischen Familien im Mutterland unmittelbar durch die Geschehnisse in den „Überseeprovinzen“, den províncias ultramarinas, betroffen. Zweitens erlebte Portugal von allen kolonialen Metropolen den stärksten Siedlerrückstrom - knapp 500.000 europäische Siedler, bald als retornados bezeichnet, stellten nach den Fluchtwellen von 1974/75 etwa 5 Prozent der portugiesischen Gesamtbevölkerung. Der Vergleich mit einem Anteil von 3,5 Prozent im Falle der aus Algerien nach Frankreich eingewanderten französischen Siedler oder mit 2,5 Prozent für den Fall der niederländischen Rücksiedler aus Indonesien zeigt bereits das Ausmaß der portugiesischen Rücksiedlungserfahrung. ${ }^{3}$ Diese Bedingungen sind verbunden mit den spezifischen Charakteristika der portugiesischen „Kolonialpraxis“, welche wir im folgenden Abschnitt betrachten werden. Sie sind verknüpft mit der besonders bruchartigen Erfahrung von Dekolonisation im portugiesischen Fall und können als fruchtbarer Boden für eine Erinnerungsdebatte angesehen werden. Umso erstaunlicher ist es angesichts dieser Rahmenbedingungen, dass eine solche Debatte praktisch gänzlich fehlt. Die gewaltsame Dekolonisation des portugiesischen Kolonialreiches im „kollektiven Gedächtnis“ ist als Referenz im Blick der gebildeten jüngeren Generationen ebenfalls wenig präsent. Zumeist wird hier die koloniale Erfahrung Portugals auf das „Zeitalter der Entdeckungen“ und den als „ruhmvoll“ betrachteten Beitrag portugiesischer Kapitäne zur Etablierung der Ostasienseerouten reduziert, die Kapitel des 20. Jahrhunderts als Ganzes werden schlicht ausgeblendet. ${ }^{4}$

Die enge Verbindung der kolonialen Situation mit der Diktatur des Estado Novo, des „Neuen Staates“ unter António Oliveira Salazar und Marcello Caetano, stellt

1 Margarida Calafate Ribeiro u. Ana Paula Ferreira, Apresentação, in: dies. (Hg.), Fantasmas e fantasias imperiais no imaginário português contemporâneo, Porto 2003, S. 9-28, hier S. $15 \mathrm{f}$.

2 Margarida Calafate Ribeiro, Uma história de regressos. Império, guerra colonial e póscolonialismo, Porto 2004, S. 246-257.

3 Rui Pena Pires, Migrações e Integração. Teoria e aplicações à sociedade portuguesa, Oeiras 2003, S. 191.

4 Rosa Cabecinhas u. João Feijó, Collective Memories of Portuguese Colonial Action in Africa. Representations of the Colonial Past among Mozambicans and Portuguese Youths, in: International Journal of Conflict and Violence 4. 2010, S. $28-44$. 
fraglos eine wichtige Besonderheit in der portugiesischen Erfahrung dar. Da eine Einordnung des Estado Novo selbst in der akademischen Debatte nur teilweise vorliegt und die Sicht der portugiesischen Bevölkerung auf die autoritäre Vergangenheit des portugiesischen Staates keineswegs so eindeutig ablehnend ist wie dies bisweilen den Anschein hat, überrascht zunächst auch der Umstand nicht, dass der späte portugiesische Kolonialstaat als Teil dieser komplizierten Bewertung wenig Diskussion erfährt. ${ }^{5}$ Zumindest die akademische Aufarbeitung anderer Themen im Zusammenhang mit dem Estado Novo hat jedoch in den vergangenen zwei Jahrzehnten an Fahrt gewonnen, während dies für die koloniale Erfahrung unter der Diktatur, einschließlich der Kolonialkriege und für den unmittelbaren Dekolonisationsprozess 1974/75 nicht der Fall ist. ${ }^{6}$ Die fehlende Beschäftigung mit der Salazar- und der Caetano-Diktatur muss somit als ein Element der Interpretation der blockierten Erinnerung an die portugiesische Dekolonisation aufgefasst werden, ist aber bei weitem nicht das einzige. Wir werden uns im Folgenden den spezifisch anderen Erklärungsansätzen zuwenden, die sich aus der Geschichte des portugiesischen Kolonialstaats und seiner Dekolonisationserfahrung ergeben.

Es ist auf den ersten Blick naheliegend, die portugiesische Erfahrung mit anderen verspäteten Debatten in europäischen Gesellschaften über die jeweilige nationale Vergangenheit zu vergleichen. Dazu wären als wichtigste Beispiele des 20. Jahrhunderts natürlich etwa der Umgang der französischen Öffentlichkeit mit dem Algerienkrieg oder auch die Auseinandersetzung in der westdeutschen Gesellschaft mit der NS-Vergangenheit zu nennen. Vor diesem vergleichenden Hintergrund erschiene die portugiesische Dekolonisationserfahrung als schmerzhafter und schuldbeladener kollektiv-nationaler Prozess in ähnlicher Form als Tabuthema. Dem ist jedoch entgegenzuhalten, dass eine portugiesische Öffentlichkeit - ganz anders als in den zuvor genannten Fällen - sehr rasch

5 Siehe zur Gedächtnispolitik in Portugal im Hinblick auf den Estado Novo António Costa Pinto, Ajustando contas com o passado na transição para a democracia em Portugal, in: Alexandra Barahona de Brito u.a. (Hg.), Política da memória. Verdade e justiça na transição para a democracia, Lissabon 2004, S. 87-108, hier S. 105-107.

6 In zwei Phasen - in den 1990er Jahren und seit 2004 - ist die historische Aufarbeitung der Salazar-Diktatur erheblich vorangeschritten. Beispiele dieser neuen Beschäftigung der portugiesischen Geschichtswissenschaft sind: João Madeira (Hg.), Vítimas de Salazar. Estado Novo e violência política, Póvoa de Santo Adrião 2007; Fernando de Castro Brandão, Estado Novo. Uma cronologia, Lissabon 2004; Fernando Rosas u. Pedro Aires Oliveira (Hg.), A transição falhada. O marcelismo e o fim do Estado Novo, 1968 - 1974, Lissabon 2004; José Carlos Valente, Estado Novo e alegria no trabalho. Uma história política da FNAT, 1935 - 1958, Lissabon 1999; Manuel Braga da Cruz, O Estado Novo e a Igreja Católica, Lissabon 1998; Susana Pereira Bastos, O Estado Novo e dos seus vadios. Contribuição para o estudo das identidades marginais e da sua repressão, Lissabon 1997. Eine erste historisch-analytische Biographie über Oliveira Salazar selbst liegt vor mit Filipe Meneses, Salazar. A Political Biography, New York 2009. 
wieder die Anknüpfung an die ehemaligen Kolonialgebiete suchte. Diese waren, sobald ihre innenpolitische Lage und politischen Regime es zuließen, willkommene ökonomische Investitionsräume und Objekte kulturpolitischer Bemühungen. Gerade im Rahmen der letzteren wäre etwa im Hinblick auf die Schaffung der Gemeinschaft der Länder portugiesischer Sprache, der Comunidade dos Paises de Língua Portuguesa (CPLP), eine Infragestellung des eigenen historischen Verhältnisses $\mathrm{zu}$ den ehemaligen Kolonien und ihren Bevölkerungen, sowie des anschließenden Bruchs, zu erwarten gewesen. ${ }^{7}$ Die Tatsache, dass trotz dieser begünstigenden Faktoren für eine Vergangenheitsdebatte die koloniale Erinnerung als öffentlicher Akt blockiert blieb, lässt einen sehr spezifischen Verlauf der Aufarbeitung kolonialer Vergangenheit plausibel erscheinen.

Wir werden dazu ein Interpretationsangebot vorlegen, welches die möglichen Ursachen der ausbleibenden Debatte im Hinblick auf Portugals koloniale Erfahrung anhand von vier Schritten diskutiert. Zunächst wird auf die spezifischen Strukturelemente des portugiesischen Kolonialreiches hingewiesen, welche eine akzeptable Erinnerungsform schwieriger auffindbar machten als etwa im Falle der britischen oder der scheinbar erfolgreichen französischen Entkolonialisierung. ${ }^{8}$ Anschließend werden wir auf die fortdauernde Aktivität zentraler kolonialer Akteure aus den letzten zwanzig Jahren des Estado Novo hinweisen, deren Präsenz im nachrevolutionären Portugal starken Einfluß auf die öffentliche Meinung ausübte. Ein drittes Kapitel nimmt die Form der gesellschaftlichen Eingliederung der retornados in den Blick, welche einer umfassenden Diskussion innerhalb Portugals entgegenwirkte. In einem letzten Schritt werden wir die spezifischen Formen der Kriegserinnerung im Rahmen einer populären Geschichtsschreibung untersuchen und zur damit in enger Verbindung stehenden akademischen Bearbeitung des Themas in Portugal zurückkehren. Letzteres betrifft namentlich die Konfrontation portugiesischer Historiker oder Populärhistoriker mit einer weitgehend von außen geprägten historischen Betrachtung der portugiesischen Kolonialrealität.

\section{Ein spezieller Fall von kolonialer Ambivalenz. Der späte portugiesische Kolonialstaat in Afrika}

In einem Großteil der afrikanischen Kolonialgebiete unter europäischer Kontrolle und für das Gros der europäischen Metropolen waren die Jahre zwischen 1951 und 1960 durch eine Teilnahme am Machttransfer gekennzeichnet. Dieser

7 Caroline Bieger-Merkli, La „Communauté des Pays de Langue Portugaise“. Un espace interculturel de coopération basé sur la langue?, Turin 2010, S. $81-112$.

8 Frank Heinlein, British Government and Decolonisation 1945-1963. Scrutinising the Official Mind, London 2002; Tony Chafer, The End of Empire in French West Africa. France's Successful Decolonization?, Oxford 2002. 
äußerte sich durch politische Reformversuche sowie durch Bemühungen, Radikalisierungsprozesse unter den afrikanischen Eliten $\mathrm{zu}$ verlangsamen, außerdem durch ein mehr oder weniger abruptes Einlenken auf einen Kurs hin zum vollständigen Machttransfer und ein ostentatives Zurschaustellen der eigenen Mitwirkung am Dekolonisationsprozess. Für das portugiesische Kolonialreich auf dem afrikanischen Kontinent lag der Fall vollständig anders. Der portugiesische „Premierminister“ und Diktator António de Oliveira Salazar und die portugiesischen Machteliten der 1950er und 1960er Jahre verweigerten gesellschaftlich-politisch Veränderungen, die zu einer graduellen Beteiligung afrikanischer Funktionäre und der portugiesisch ausgebildeten Elite an der politischen Macht geführt hätten. Im portugiesischen Überseeministerium (Ministério do Ultramar) - wie das einstige Kolonialministerium seit 1951 bezeichnet wurde - ist für die gesamten 1950er Jahre keinerlei Planung für eine etwaige Reform der politischen Strukturen des Kolonialregimes zu erkennen. Im Gegenteil, die Kolonialgebiete wurden in der portugiesischen Rhetorik zunehmend als Teilprovinzen des Mutterlandes behandelt, obschon diese Wortwahl keine konkreten Folgen auf lokaler Ebene hatte. ${ }^{9}$ Zugleich wurde, mit einem Höhepunkt in der ersten Jahreshälfte 1961 nach den Revolten in Angola, die Idee des portugiesischen Kolonialreiches als einer „lusotropischen“, „multirassischen" und harmonischen Gesellschaft stark gemacht. Von diesbezüglichen Rechten im Sinne einer Teilhabe an der Kolonialgesellschaft profitierte jedoch allenfalls die verschwindend kleine afrikanische und gemischte „Mittelschicht“ in den Kolonien, während die entsprechende Rhetorik für die große Bevölkerungsmehrheit keinerlei Relevanz hatte. Daran änderte sich bis zur portugiesischen Nelkenrevolution von 1974 letztlich wenig. ${ }^{10}$

Es kann hier nicht im Detail diskutiert werden, ob die portugiesische Form des späten Kolonialstaates im subsaharischen Afrika tatsächlich in allen Aspekten so grundverschieden war von den Strukturen der anderen europäischen Kolonialregime. ${ }^{11}$ Zweifel sind berechtigt, stand doch die potentielle Einbindung der jeweiligen Bevölkerungen und vor allem von lokalen Eliten in die Verwaltungsstrukturen vor durchaus ähnlichen Herausforderungen. ${ }^{12}$ Dabei entsprach die Grenzziehung freilich der autoritären Natur des Regimes im Mutterland: Wo in Portugal selbst die Einführung zumindest semi-demokratischer Ansätze bis zu den Wahlen von 1958 nur schleppend voranging, war eine Schaffung demokra-

9 Für einen Überblick der propagandistisch-rhetorischen Formen vgl. Ribeiro, História de Regressos, S. $117-138$.

10 Malyn Newitt, Portugal in Africa. The Last Hundred Years, London 1981, S. 138 - 142.

11 Die neueste vergleichende Betrachtung der Dekolonisationserfahrungen in Europa findet sich in Martin Thomas, Contrasting Patterns of Decolonization. Belgian and Portuguese Africa, in: Martin Thomas u. a. (Hg.), Crises of Empire. Decolonization and Europe's Imperial States, 1918 - 1975, London 2008, S. 385 -410, hier S. $393-410$.

12 Alexander Keese, Living with Ambiguity. Integrating an African Elite in French and Portuguese Africa, 1930 - 1961, Stuttgart 2007. 
tischer Institutionen in den Kolonialgebieten schwerlich vorstellbar. Liberalisierungsprozesse finden sich durchaus im Hinblick auf die Professionalisierung von Verwaltungsstrukturen - denn die Missbräuche gegenüber kolonialen Bevölkerungen gingen in den 1950er Jahren rapide zurück und der Einsatz von afrikanischen Kolonialangestellten in den (zumeist subalternen) Funktionen der kolonialen Verwaltung wurde zunehmend typischer. Auch dieser Liberalisierungsprozess war von Widersprüchen durchsetzt. So blieb das brennendste Problem in den Lebensrealitäten der lokalen Bevölkerungen, die Existenz von staatlich verordneter Zwangsarbeit und Körperstrafen unter einem spezifischen Gesetzeskodex für die „indigene“ Bevölkerung. Während er in den anderen Kolonialreichen in den 1940er Jahren abgeschafft wurde, blieb er im portugiesischen Kolonialreich, auch in der Variante des späten Kolonialstaates, bis 1961/ 62 erhalten. ${ }^{13}$ Bereits vor Beginn der antikolonialen Aufstände in Angola wuchs unter dem Einfluss mehrerer unabhängig voneinander agierender Gruppen liberaler Verwaltungsbeamter und Kolonialinspektoren - die ihre Ideen teilweise bis auf die höchsten Ebenen des Überseeministeriums tragen konnten - der Druck im Hinblick auf eine Verbesserung der Lebensbedingungen des Gros der afrikanischen Bevölkerungen in den „Überseeprovinzen“. Dieser Druck nahm zu und hatte eine Reihe konkreter Verbesserungen zur Folge, als zwischen 1961 und 1964, parallel zur Ausweitung der Aufstände gegen die Kolonialmacht, das portugiesische Militär die Verwaltung der Kolonialgebiete zunehmend mitzubestimmen begann. Schon aus strategischen Gründen wurde eine erhebliche Verbesserung der sozialen Infrastruktur angestrebt, die sich in der Schaffung von Gesundheitsposten und Schulen in ländlichen Regionen niederschlug. Es wäre vor diesem Hintergrunde falsch, den späten portugiesischen Kolonialstaat als ein Gebilde anzusehen, dessen Verwaltungseliten ausschließlich auf Repression setzten. ${ }^{14}$

Dem grundsätzlichen Willen zu Veränderungen, den zahlreiche Verwaltungsbeamte zeigten, stand allerdings ein anderes Element der kolonialen Zielsetzungen geradezu diametral entgegen. Über den gesamten Zeitraum seit Beginn der verstärkten kolonialen Kontrolle Portugals im letzten Viertel des 19. Jahrhunderts hatten portugiesische Regierungen den Nutzen einer europäischen Besiedlung gerade der großen Territorialgebiete betont. Die Erfolge hielten sich in dieser Hinsicht allerdings in Grenzen. Erst nach dem Zweiten Weltkrieg beschleunigte sich die Einwanderung portugiesischer Siedler nach Angola und Mosambik. Ab den 1950er Jahren nahm sie dann in extremem Maße zu und führte $\mathrm{zu}$ einer starken Veränderung der sozialen Zusammensetzung der Bevölkerungen in den beiden flächenmäßig größten „Überseeprovinzen“. Die

13 Malyn Newitt, The Late Colonial State in Portuguese Africa, in: Itinerario 22. 1999, S. $110-122$.

14 Alexander Keese, Dos abusos às revoltas? Trabalho forçado, reformas portuguesas, política „tradicional“ e religião na Baixa de Cassange e no Distrito do Congo (Angola), 1957 - 1961, in: Africana Studia 7. 2004, S. $247-276$, hier S. $257-260$. 
Hoffnung des Salazar-Regimes auf eine agrarische Erschließung des ländlichen Hinterlandes der Kolonien erfüllte sich nur in einzelnen Regionen, und ein erheblicher Teil der europäischen Immigranten konzentrierte sich in den urbanen Räumen: die Zentren von Luanda und Lourenço Marques (des heutigen Maputo) wurden regelrecht zu „weißen“ Städten. ${ }^{15}$ Die Präsenz der Siedler bremste jegliche rasche Reform - die liberalen Teile der Kolonialverwaltung konnten sich mit ihren Sichtweisen hinsichtlich sozialer Verbesserungen, Veränderungen in der Rechtsstellung der kolonialen Untertanen und Infrastrukturprojekten zum Vorteile vor allem der schwarzafrikanischen Bevölkerungen nur schleppend durchsetzen. Ihnen konnte stets entgegengehalten werden, ihre Positionen gefährdeten das Prestige und die Wohlfahrt der europäischen Siedler.

Während des Kolonialkrieges sollte Portugals autoritäres Regime heftigen Anfeindungen vor den Vereinten Nationen und in anderen internationalen Gremien ausgesetzt sein. Vielfach wurde die „Rückständigkeit“ der portugiesischen Kolonialprogramme attackiert, Portugal so als ein nicht nur reaktionärer, sondern auch zu größeren sozialen Leistungen unfähiger Staat dargestellt. ${ }^{16}$ Natürlich wurden die Vertreter der neuen afrikanischen Staaten und der blockfreien Bewegung besonders durch die portugiesische Rhetorik als „koloniales Problem“ entworfen. Die Haltung der portugiesischen Machteliten, die Kolonien als unverzichtbare Teile des Mutterlandes zu behandeln und an dieser Position unbeirrt festzuhalten, war grundsätzlich verschieden von den Dekolonisationswegen anderer europäischer Mutterländer und für die Gegner Portugals in den internationalen Institutionen völlig inakzeptabel. Zudem ließen die Informationen über die portugiesische koloniale Organisation ihre Kolonialgebiete im - zumal oberflächlichen - Vergleich als besonders schlecht verwaltet und extrem repressiv regiert erscheinen.

Der späte portugiesische Kolonialstaat war damit besonders in sich widersprüchlich. Ähnlich wie in Südrhodesien und in Südafrika wirkte die immense Präsenz der Siedler jeglicher rascher Reform zugunsten der schwarzafrikanischen Bevölkerungen entgegen; die autoritäre Regierung in Lissabon wurde zur Zielscheibe wütender Kritik aus aller Welt. Bereits unter dem Estado Novo bemühte man sich, diese Widersprüche aus der Diskussion auszublenden. Die Aufständischen seien sowjetisch inspirierte „Terroristen“, die die Vorzüge der „multirassischen“, egalitären Ausrichtung der portugiesischen Kolonialpolitik schlicht nicht verstünden. Als sich die ersten Wogen der nachrevolutionären Begeisterung zugunsten einer raschen Dekolonisation zumindest von Angola

15 Christine Messiant, Luanda $1945-1961$. Colonisés, société coloniale et engagement nationaliste, in: Michel Cahen (Hg.), „Vilas“ et „cidades“. Bourgs et villes en Afrique lusophone, Paris 1989, S. 125-199; Jeanne-Marie Penvenne, African Workers and Colonial Racism. Mozambican Strategies and Struggles in Lourenço Marques, 1877 - 1962, London 1995.

16 John D. Hargreaves, Decolonization in Africa, London 1988, S. $205-218$. 
und Mosambik geglättet hatten, war 1974/75 ein kurzer Versuch von Seiten der neuen politischen Kräfte im Mutterland zu einer Neubewertung der kolonialen Erfahrung zu kommen, beendet, bevor er wirklich begonnen hatte. Stattdessen kehrte der Umgang mit den Widersprüchen der kolonialen Vergangenheit, nun schon in der Retrospektive, in die bewährten unkritischen Bahnen zurück.

\section{Von Kolonialministern zu Größen in Portugals akademischer Welt. Adriano Moreira, Joaquim da Silva Cunha und die Banalisierung der Repression}

Die Liberalisierung portugiesischer Kolonialpolitik in den 1950er Jahren führte dazu, dass als relativ reformorientiert und liberal geltende Personen, zum Teil von eigenen Vertrauensleuten flankiert, auf die wichtigsten administrativen Posten des Kolonialreiches gelangten. Dies galt zum Teil für die Generalgouvernements von Angola und Mosambik und dann vor allem für das Kolonialbeziehungsweise seit 1951 Überseeministerium selbst. Bereits Sarmento Rodrigues, der 1951 das Ruder des Ministeriums übernommen hatte, hatte einen vergleichsweise liberalen Kurs verfolgt. Seine Nachfolger setzten diese Ausrichtung fort - Adriano Moreira, Überseeminister von 1961 bis 1962, sowie Joaquim Moreira da Silva Cunha, auf derselben Position von 1965 bis 1973, genossen die Reputation, den reformerischen Kräften innerhalb der portugiesischen Diktatur anzugehören.

Diese Zuschreibung war nicht vollständig unberechtigt, wie sich an einer Reihe bisher nicht analysierter Korrespondenzen der späten 1950er Jahre verdeutlichen lässt. Joaquim da Silva Cunha, Professor am Centro de Estudos Políticos e Sociais der Junta de Investigações do Ultramar in Lissabon und beständiger Korrespondenzpartner des Überseeministeriums in den 1950er Jahren, trat etwa als Unterstützer des besonders liberalen Distriktgouverneurs des KongoDistriktes im nördlichen Angola hervor. Letzterer suchte bereits zwischen 1956 und 1957 in Eigeninitiative Zwangsarbeit durch freie Arbeitsverträge abzulösen, und Silva Cunha stellte sich auf seine Seite. ${ }^{17}$ Gemeinsam mit Moreira, der bis 1959 Direktor des genannten Zentrums war, und einer Gruppe reformbereiter Kolonialfunktionäre ermahnte Silva Cunha in einem Bericht von 1959 den Überseeminister Vasco Lopes Alves zur dringenden Umsetzung notwendiger Reformen im Hinblick auf die Arbeitsbedingungen der afrikani-

17 Arquivo Histórico Ultramarino, Lissabon, Portugal (AHU), MU/GM/GNP/135/Cx. 35, Esteves Felgas, Distriktgouverneur des Congo, Angola, Distrito do Congo: Relatório, 1957 - Elementos pedidos pelo Exm ${ }^{\circ}$ Professor Joaquim Silva da Cunha [1957]. Zur Pionierrolle von Esteves Felgas siehe auch Alexander Keese, „Proteger os pretos“. Havia uma mentalidade reformista na administração portuguesa da África tropical, 1926-1961?, in: Africana Studia 6. 2003, S. $97-125$, hier S. $108-110$. 
schen Bevölkerungen. ${ }^{18}$ Die Kritik, die in diesem Bericht formuliert wurde, war so scharf, dass das Schriftstück Premierminister Oliveira Salazar selbst vorgelegt wurde, der äußerst irritiert darauf reagierte. ${ }^{19}$ Die im Vergleich liberale koloniale Grundhaltung der beiden künftigen Ministerpersönlichkeiten darf freilich nicht darüber hinwegtäuschen, dass sie dennoch loyale Anhänger des Regimes und der meisten Grundlinien seiner Kolonialpolitik waren, einschließlich der diskriminierenden Rechtslage der afrikanischen Bevölkerungen. Moreira insbesondere spielte seit 1958 in der Gegenpropaganda des Kolonialregimes vor dem Hintergrund panafrikanischer antikolonialer Bewegungen und Institutionen eine wichtige Rolle. ${ }^{20}$ Silva Cunha vertrat im Hinblick auf Reformforderungen ursprünglich die weitergehenden Positionen im Vergleich zu Moreira, zeigte jedoch in Publikationen über die Arbeitshaltung der „Indigenen“ ebenfalls klare Grenzen seiner Liberalität. Ob er wirklich an irgendeinem Punkt für eine durchgehende Beseitigung von Zwangsarbeit und diskriminierenden Rechtsnormen eintrat, muss als sehr fraglich angesehen werden. ${ }^{21}$

Die Aktivität beider Personen im Ministeramt blieb in der Praxis weitaus weniger engagiert, als dies ihre oftmals kritischen Einwürfe der späten 1950er Jahre hätten vermuten lassen. Moreira hatte den Vorteil, die schon über Jahre diskutierte und angemahnte Abschaffung von Zwangsarbeit und den schärfsten rechtlichen Diskriminierungen der lokalen Bevölkerungen im Kolonialreich endlich umsetzen zu können. Zugleich und vor allem sorgte er jedoch für eine rasche Ausarbeitung einer schlagkräftigeren Kolonialpropaganda, welche Themen der "gemischten portugiesischen Gesellschaft“ aufgriff. Die entsprechenden Denkströmungen waren während des ganzen 20. Jahrhunderts immer wieder zum Gegenstand intellektueller Debatten über den „portugiesischen Nationalcharakter" geworden, ohne freilich systematisch und als politisches Konzept betont $\mathrm{zu}$ werden. ${ }^{22}$ Unter Moreira änderte sich dies. Stärkster

18 Arquivo Nacional da Torre do Tombo (ANTT), AOS/CO/UL-61, Relatório [do Centro de Estudos Políticos e Sociais, Lissabon] an Vasco Lopes Alves, portugiesischer Überseeminister [1959], S. $11 \mathrm{f}$.

19 ANTT, AOS/CO/UL-61, Gesundheitsministerium (Ministério da Saúde e Assistência) an Oliveira Salazar, portugiesischer Premierminister, 2.9.1959.

20 AHU, MU/GM/GNP/084/Cx. 33, Adriano Moreira, Direktor des Centro dos Estudos Políticos e Sociais der Junta das Investigações do Ultramar, Lissabon, an Direcção Geral de Administração Política e Civil (Nr. 1468/47 A/58), 4.9.1958, S. 2; AHU, MU/GM/ GNP/084/Cx. 33, Portugiesisches Überseeministerium, Direcção Geral de Administração Política e Civil, Despacho - Organização de uma contra-propaganda efectiva tendente a evitar ou desfazer críticas infundadas à nossa política ultramarina, 1.7. 1959, S. 1.

21 Joaquim Moreira da Silva Cunha, O sistema português de política indígena. Princípios gerais, Lissabon 1952.

22 Miguel Bandeira Jerónimo, Livros Brancos, Almas Negras. A „missão civilizadora“ do colonialismo português, 1870-1930, Lissabon 2009, S. 251-268; Patrícia Ferraz de 
Ausdruck der neuen Aktivität war die Einladung an den einflussreichen brasilianischen Soziologen Gilberto Freyre, bekannt durch seine Schriften zu den Vorzügen einer "gemischtrassigen“" Gesellschaft. ${ }^{23}$ Freyre wurde auf Anordnung und unter Mitwirkung von Moreira stark von portugiesischen Verwaltungsbeamten hofiert. Tatsächlich sollte der anerkannte Soziologe nach einer Reise durch die portugiesischen Territorien auf dem afrikanischen Kontinent zu dem Schluss kommen, die portugiesische Regierung praktiziere jenes Prinzip, das er auch für Brasilien angemahnt hatte, nämlich eine Politik der vorurteilslosen Einbindung von Gruppierungen aller Hautfarben. ${ }^{24}$ In der Praxis blieb der Prozess jedoch nach der Umsetzung jener Reformen, die schon seit Jahren diskutiert worden und gewissermaßen ein Erbe der Aktivität von Moreiras Vorgängern waren, fern von weitergehenden Reformbemühungen. Als Adriano Moreira 1962 das Amt verließ, waren die Resultate seiner Amtsführung weitaus mehr von Repression in Angola und den anderen Kolonien als von wirklichen Veränderungen geprägt. Silva Cunhas achtjährige Amtszeit bis 1973 war noch weniger innovativ. Der Überseeminister hatte im Wesentlichen auf den Druck der portugiesischen Militärs in Angola, Mosambik und Guinea-Bissau zu reagieren. Im Falle der beiden letzteren Gebiete versuchten die jeweiligen Kommandanten die problematische militärische Situation durch eine Gewinnung der Unterstützung ländlicher Bevölkerungen über soziale Maßnahmen zu verbessern. ${ }^{25}$ In Angola dienten soziale Programme im Hinterland der von europäischen Siedlern dominierten Stadtmetropolen dazu, die erfolgreiche militärische Strategie, welche eine Marginalisierung der antikolonialen Rebellengruppen erlaubt hatte, zu begleiten. ${ }^{26}$ Von einer aktiven Sozialpolitik seitens des Überseeministeriums, wie sie sich in den 1950er Jahren - obschon noch mit bescheidenen Resultaten - angekündigt hatte, kann für die Periode der antikolonialen Guerilla nicht die Rede sein.

Nach dem 25. April 1974 schienen die politischen wie universitären Karrieren von Silva Cunha und Moreira beendet zu sein. Die ersten 18 postrevolutionären Monate sahen erhebliche Bemühungen, die Kader der Diktatur aus Positionen zu drängen; Moreira entzog sich diesen Entwicklungen unmittelbar durch seine Flucht nach Brasilien, Silva Cunha wurde zwischenzeitlich inhaftiert und reiste

Matos, As côres do império. Representações raciais no império colonial português, Lissabon 2006, S. 68 - 93.

23 Cláudia Orvalho Castelo, „O modo português de estar no mundo“. O luso-tropicalismo e a ideologia colonial portuguesa, 1933 - 1961, Porto 1998, S. $62-65$.

24 Yves Léonard, Salazarisme et lusotropicalisme, histoire d'une appropriation, in: Lusotopie 1997, S. 211 -226; Ribeiro, História de Regressos, S. 151 - 166.

25 Mustafah Dhada, Warriors at Work. How Guinea Was Really Set Free, Niwot 1993, S. $38-41$.

26 John P. Cann, Counterinsurgency in Africa. The Portuguese Way of War, 1961 - 1974, London 1997. 
dann ebenfalls in den südamerikanischen Staat aus. ${ }^{27}$ An diesem Punkte wäre es kaum zu erwarten gewesen, dass die beiden Politiker noch einmal im Rampenlicht erscheinen oder gar die Interpretation von Portugals spätkolonialer Vergangenheit mitbestimmen könnten.

Das Blatt wendete sich jedoch erstaunlich rasch. Adriano Moreira kehrte bereits 1976 nach Portugal zurück und wurde als Deputierter in der Nationalversammlung (Assembleia Nacional) unter dem Schirm des rechtskonservativen Centro Democrático Social (CDS) auch wieder in die politische Öffentlichkeit integriert. Er wirkte zugleich als hochdekorierter Professor des Instituto Superior de Ciências Sociais e Políticas an der Universidade Técnica in Lissabon, und erlangte 2007 und 2009 mit seiner jeweiligen Wahl zum Präsidenten der Akademie der Wissenschaften in Lissabon eine der höchsten wissenschaftlichen Auszeichnungen in Portugal. Silva Cunhas Weg zurück in die „Respektabilität“ im postrevolutionären Portugal verlief weniger gradlinig. Er argumentierte aus dem brasilianischen Exil 1977, er sei, obschon Architekt des sozialen Fortschritts im Ultramar, erbarmungslos verfolgt worden. ${ }^{28}$ Doch Mitte der 1980er Jahre eröffnete sich auch für Silva Cunha der Rückweg; als Rechtsprofessor und Herausgeber verschiedener Fachzeitschriften an der privaten Universidade Portucalense in Porto etablierte er sich erneut im akademischen Establishment, wenngleich er sich nie vollständig in den politischen Strukturen des nachrevolutionären Portugals wiederfand. ${ }^{29}$

Eine empirisch fundierte Analyse der individuellen Positionen von Silva Cunha und Moreira nach ihrer Rückkehr und innerhalb der nun von ihnen neu wahrgenommenen Ämter ist schwierig bis unmöglich. Es ist durchaus denkbar, dass beide sich individuell von ihren noch in den 1960er und 1970er Jahren vertretenen Sichtweisen distanziert haben. ${ }^{30}$ Wichtiger für die in Portugal geführte Auseinandersetzung mit der spätkolonialen Vergangenheit und der Dekolonisation ist freilich, dass beide in wissenschaftlicher, populärwissenschaftlicher und medienintensiver Weise weitgehend unkritische Positionen bedient haben. Silva Cunha, der an seiner Universität als Herausgeber der Zeitschrift Africana auch Einfluss auf die portugiesischsprachigen Debatten über die koloniale Vergangenheit nehmen konnte, zeigt dies geradezu paradig-

27 Pinto, Ajustando contas, S. $104 \mathrm{f}$.

$28 \mathrm{Zu}$ seinen sozialen Programmen, aus der Eigenwahrnehmung im brasilianischen Exil, siehe Joaquim da Silva Cunha, O Ultramar, a Nação e o „25 de Abril“, Coimbra 1977, S. $207-217$.

29 Vgl. Fernanda Paula Fernandes Oliveira de Sousa Maia, Tábua cronológica das etapes marcantes da vida profissional e académica do Professor Doutor Joaquim Moreira da Silva Cunha, in: Estudos de Homenagem a Joaquim M. da Silva Cunha, Porto 1999, S. 15-21; Jorge Miranda (Hg.), Estudos em Homenagem ao Prof. Doutor Joaquim Moreira da Silva Cunha, Coimbra 2005.

30 Anhaltspunkte widersprüchlicher Art finden sich in Adriano Moreira, A espuma do tempo. Memórias do tempo de vésperas, Coimbra 2009. 
matisch in einem Aufsatz „Wie wir die Überseeprovinzen regierten“. In dieser Publikation fehlt jegliche Kritik, sie beschränkt sich auf eine absolut technische Betrachtung der portugiesischen Kolonialorganisation. ${ }^{31}$ Moreira seinerseits gibt heute $\mathrm{zu}$, dass eine Verhandlungslösung zur Dekolonisation eventuell die bessere Herangehensweise gewesen wäre, aber es fehlt auch von seiner Seite jede kritische Auseinandersetzung mit den repressiven Strukturen, die das Kolonialreich so sehr geprägt haben. ${ }^{32}$ Auf der anderen Seite wurde von Moreira die Beziehung Portugals zum subsaharischen Afrika über andere Verbindungslinien stark gemacht. Er bezog sich etwa auf Portugals Verpflichtung zu einer Zusammenarbeit mit den Kapverdischen Inseln, die für ihn gar in die Europäische Union aufzunehmen wären, unter tatkräftiger Zusammenarbeit der alten Kolonialmacht Portugal mit den neuen Eliten der ehemaligen Kolonie. $^{33}$

Die Tatsache, dass zwei dem Regime des Estado Novo verbundene, führende Kolonialpolitiker und Denker zu kolonialen Strategien, zumindest bis in die 1990er Jahre hinein - wenn nicht bis zum heutigen Tag - den Diskurs über den portugiesischen späten Kolonialstaat maßgeblich mit besetzt haben, beeinflusste die Auseinandersetzung mit dieser Vergangenheit, und ist zugleich bezeichnend für das Herangehen an die Thematik in Portugal. Es muss an dieser Stelle wiederholt werden, dass Adriano Moreira und Joaquim da Silva Cunha in den 1980er und 1990er Jahren nicht offen als Apologeten derjenigen repressiven kolonialen Praktiken auftraten, für welche sie in leitenden Funktionen mit verantwortlich gewesen waren. Sie trugen zum Diskurs beschreibende Beiträge und eine paternalistische Position bei, die durchblicken ließ, dass man sehr früh auf Reformen im Kolonialreich hingewirkt hatte. Selbstkritik oder überhaupt eine kritische Distanzierung von den Strukturen kolonialer Beherrschung fehlen hingegen. Die spezifische Natur dieser Haltungen - im Unterschied zu scheinbar ähnlichen Karrieren wie zum Beispiel jener des französischen Gouverneurs und Generalgouverneurs Pierre Messmer, der es unter der Präsidentschaft Charles de Gaulles in den 1960er Jahren zum Kriegsminister brachte und unter der Präsidentschaft Georges Pompidous ab 1972 sogar zum Premierminister - liegt darin begründet, dass in Portugal auf der innenpolitischen und gesellschaftlichen Ebene durch die Revolution vom 25. April 1974 ein Bruch stattgefunden hatte, während im Hinblick auf das Ultramar die Sprecherrolle Moreiras und Silva Cunhas eine Kontinuität suggerierte. Letzteres Phänomen bleibt singulär für eine frühere Kolonialgesellschaft. Es spielt eine wichtige Rolle für das Verständnis des portugiesischen Erinnerungsprozesses.

31 Joaquim da Silva Cunha, Como governámos o Ultramar. Do demo-liberalismo à descolonização, in: Africana 8. 1991, S. 5-38.

32 Adriano Moreira, Era possível uma solução política. Entrevista de Adelino Gomes, in: Público, Dossier „E podia ter sido de outra maneira? 1975-1995. Vinte anos de independências“, 22.4.1995, S. 4.

33 Ders., A integração de Cabo Verde na Europa, in: Diário de Notícias, 8. 2.2005, S. 17. 


\section{Die retornados. Eine Integration ohne soziale Eruption}

Der Exodus wie auch die soziale Integration der portugiesischen Siedler, die unerwartet rasch und scheinbar friedlich verliefen, unterscheiden sich von der Rückkehr anderer Siedlerbevölkerungen in die europäischen „Mutterländer“; diese waren oftmals mit erheblichen sozialen Spannungen verbunden, die sich teilweise bis in die Gegenwart wahrnehmen lassen, während sich ähnliche Frontstellungen im portugiesischen Fall nicht oder kaum finden. Die Präsenz der aus den Kolonien zurückkehrenden Siedler, die es in anderen europäischen Staaten so schwierig, aber zugleich auch umso notwendiger machte, eine Erinnerungsformel für die koloniale Vergangenheit und vor allem für den Dekolonisationsprozess zu suchen, gestaltete sich im portugiesischen Fall weit weniger als gesellschaftlicher Sprengstoff.

Ein knapper vergleichender Blick illustriert das Außergewöhnliche dieser portugiesischen Erfahrung. Die ökonomische und demographische Dimension der Besiedlung von Kolonialterritorien machte im Prinzip besonders Italien, Frankreich und Portugal anfällig für soziale Verwerfungen im Moment einer Dekolonisation, die mit einer erzwungenen „Repatriierung“ einherging. Im italienischen Fall handelte es sich um immerhin 600.000 Siedler, unter denen zumal kaum qualifizierte Arbeiter waren. Ihre Rückkehr fand schrittweise statt, was ihnen jedoch weder die auf der Apenninenhalbinsel der 1950er Jahre endemische Arbeitslosigkeit noch die feindselige Bemitleidung von Seiten ihrer Landsleute ersparte. Für einen gewissen Zeitraum herrschten bei den ehemaligen Siedlern Zorn und Frustration vor, die sich in ihren Verbänden und in der beständigen Propaganda der Neofaschistischen Partei Movimento Sociale Italiano (MSI) kanalisierten; die letztere wurde zum Sprachrohr der Siedler. Die französischen pieds-noirs fanden im Gegensatz dazu 1962 ein Mutterland vor, welches durch ökonomische Expansion und einen Bedarf an Arbeitskräften charakterisiert wurde. Diese Ausganglage erleichterte ihre Einbindung, gleichzeitig sorgten ihre schiere Zahl von circa einer Million und ihre Orientierung zur politisch extremen Rechten dafür, dass sie ein schwer zu handhabender Teil der Bevölkerung blieben. Für die portugiesische Rücksiedlung aus den Kolonialterritorien war der Fall grundsätzlich noch problematischer, denn wie bereits festgestellt, war die Zahl der Siedler immens und das Mutterland ganz besonders in den Jahren 1975 und 1976 von Armut, zusätzlichen Auflösungserscheinungen aufgrund der ökonomischen Krise und starken politischen Turbulenzen geprägt. $^{34}$

Dennoch lassen sich für den Prozess der Integration der portugiesischen Siedler in die Gesellschaft des Mutterlandes keine nachhaltig wirksamen sozialen Spannungen oder Zwischenfälle feststellen. Unter den retornados als Gruppe

34 Bernard Droz, Pieds-noirs, Belges, Anglais, Portugais... Le grand rapatriement, in: L'Histoire 231. 1999, S. 70 - 73, hier S. 73. 
existierten zweifellos zu spezifischen Zeitpunkten Äußerungen von Solidarität und Zusammenhalt. Eine wirklich geschlossene Gemeinschaft mit eigenständiger Identität stellten sie jedoch auf portugiesischem Boden nicht dar, und über relativ wenige Jahre hinweg taten der gemeinsame Schulbesuch beziehungsweise die Ausbildung, der Kontakt am Arbeitsplatz oder das Zusammenwohnen mit der Bevölkerung der Metropole ihr Übriges, um die Einbindung der retornados in die Tat umzusetzen. ${ }^{35}$ Damit kann der portugiesische Fall mit seinen spezifischen Charakteristiken tatsächlich als vollständig außergewöhnlich für einen Rückkehrprozess von kolonialen Siedlern betrachtet werden: die Verbindung einer späten, unerwarteten und damit völlig ungeplanten Dekolonisation mit einer schwierigen wirtschaftlichen Konjunkturlage, einer instabilen politischen und sozialen Situation im Mutterland in der Folge der Revolution, einer Konzentration des Rückstroms der Siedler auf einen extrem kurzen Zeitraum, und einer erheblichen Zahl an Individuen im Verhältnis zu Bevölkerungszahl und Möglichkeiten des Aufnahmelandes lassen die vergleichsweise konfliktarme soziale Integration dieser Siedlergruppe in der Tat als ungewöhnlich und erklärungsbedürftig erscheinen.

An dieser Stelle spielt zunächst einmal eine wichtige Rolle, dass nicht nur die Dekolonisation, sondern auch die Besiedlungsgeschichte der portugiesischen Flächenkolonien im subsaharischen Afrika chronologisch ganz entscheidend von den Prozessen der anderen Kolonialmächte abweicht. Während die Rückkehrbewegungen europäischer Siedler im Falle aller anderen kolonialen Mutterländer sich vor allem zwischen dem Ende des Zweiten Weltkrieges und den frühen 1960er Jahren häuften, war dies exakt der Zeitraum, in welchem sich, wie oben diskutiert, die Wanderungsbewegungen portugiesischer Siedler nach Angola und Mosambik überhaupt erst intensivierten. Spätestens seit den frühen 1960er Jahren sollten diese Siedler unter der Ägide von Überseeminister Adriano Moreira - trotz aller reellen negativen Effekte auf die Reformpolitik in den portugiesischen Kolonien, die wir oben beschrieben haben - Teil der neuen portugiesischen Kolonialpropaganda werden. Sie galten in der Propaganda als Teil einer „rassenharmonischen“ Besiedlung der afrikanischen Territorien. Als paradoxer Nebeneffekt führte diese Rhetorik auch dazu, dass die Rückkehrbewegung ab 1975 „ethnisch“ deutlich heterogener war als im Falle anderer Kolonialreiche - schwarzafrikanische und "gemischte“ (mestiço) Bevölkerungen in Angola und Mosambik mit portugiesischem Pass konnten von der Immigration ins „Mutterland“ nicht ausgeschlossen werden. Dies sorgte für eine frühe Entstehung afrikanischer Gemeinschaften auf dem Boden Portugals. ${ }^{36}$

Der Prozess der „Repatriierung“ der retornados verlief dann relativ schlagartig. Oberflächlich schien er durch die Haltungen der neuen portugiesischen

35 Rui Pena Pires u.a., Os retornados. Um estudo sociográfico, Lissabon 1984, S. 23.

36 Fernando Dacosta, Os retornados, in: João Medina (Hg.), História de Portugal, Bd. 14, Lissabon 1998, S. $223-230$, hier S. 224. 
Regierungen im Anschluss an die Revolution bestimmt zu werden, doch in der Realität reagierten diese Regierungen lediglich auf äußere Zwänge. Die antikolonialen Bewegungen, die in der Folge des 25. April 1974 in Angola und Mosambik die politische Macht übernahmen beziehungsweise - in Angola - um diese Macht erbittert kämpften, diktierten letztlich den Verlauf des Prozesses. ${ }^{37}$ Im Gegensatz zu anderen Kolonialmächten hatten die portugiesischen Regierungen damit nur geringe Möglichkeiten, die Entwicklung zu lenken; die entscheidenden Rückführungsaktionen hatten in kurzen Zeiträumen zu geschehen. ${ }^{38}$ Dieser Verlauf hat eine gewisse Ähnlichkeit mit der französischen Erfahrung in Algerien, aber der Druck, unter dem staatliche Akteure zu agieren hatten, war letztlich im portugiesischen Fall noch größer. Zeitgenossen bewerteten die Bedingungen als „chaotisch und traumatisch für Portugal“. In der Tat standen die Zeichen für die portugiesische Gesellschaft vor diesem Hintergrunde auf Sturm - die Gefahr, dass der „üble Wind“ aus den Kolonien, wie ihn die Mitglieder der regierenden Junta de Salvação Nacional (Komitee zur Nationalen Rettung) bezeichneten, einmal mehr die neue, post-rechtsautoritäre Regierung in Schwierigkeiten bringen könnte, schien sich zu erfüllen. Die Konfrontation mit einer unzufriedenen Siedlergemeinschaft wurde als ernste Gefahr gesehen. ${ }^{39}$ Dazu kam, dass die Sprecher der Flüchtlinge, die sich in Hilfskomitees organisiert hatten, den Eindruck erhielten, von den postrevolutionären Regierungen und der neuen Armeeführung im Stich gelassen zu werden. Zum Eklat kam es, als am 24. und 25. September 1975 die Mitglieder eines solchen Hilfskomitees nach Morddrohungen vom Flughafen Nova Lisboa in Angola ausgeflogen wurden, weil der portugiesische Kommandant sich nicht bereit erklärte, ihren Schutz zu garantieren. ${ }^{40}$ Diese Situation, sicherlich bedingt durch den Kontrollverlust der portugiesischen Regierungen über den Dekolonisationsprozess, ließ unter vielen der Siedler zumindest zeitweise Wut und Bitterkeit zur dominierenden Haltung gegenüber dem Mutterland werden. Trotz dieser anfänglichen Probleme erlaubte es eine Reihe von Faktoren, dass der portugiesische Fall der Einbindung kolonialer Rücksiedler günstiger verlief als in Italien oder Frankreich. Dafür sorgte zunächst einmal das Profil der Rückwanderer. Sie waren in ihrer Mehrheit Emigranten der ersten Generation, vergleichsweise jung, männlich und hatten ein Bildungsniveau, welches über

37 Norrie MacQueen, The Decolonization of Portuguese Africa. Metropolitan Revolution and the Dissolution of Empire, London 1997, S. 215 f.

38 Gonçalves Ribeiro, A vertigem da descolonização, Lissabon 2002, S. 377.

39 Norrie MacQueen, An Ill Wind? Rethinking the Angolan Crisis and the Portuguese Revolution, 1974-1976, in: Itinerario 25. 2002, S. $22-44$.

40 AHU, Arquivo do Ex IARN, 264, Carlos Alberto Marques Pinto Pereira, Koordinator; Fernando Mário Trindade Mira Godinho u.a., für Comissão Executiva de Rapatriamento da Comissão Nacional de Apoio aos Deslocados [Exekutivkommission für Repatriierung des Nationalen Hilfskomitees für Vertriebene] in Nova Lisboa, an Gabinete de Angola, Presidência da República [29.9.1975]. 
demjenigen der portugiesischen Bevölkerung des Mutterlandes lag. ${ }^{41}$ Trotz ökonomischer Stagnation in Portugal fiel die Rückkehrbewegung mit tiefgehenden Anpassungsprozessen des portugiesischen Arbeitsmarktes zusammen, welche die Integration der retornados erleichterten. Der öffentliche Sektor wuchs in der zweiten Hälfte der 1970er Jahre in Portugal ganz erheblich, und zugleich vollzog sich ein Rückgang des traditionellen Kleinunternehmertums, der wiederum den Eintritt und die unternehmerische Initiative neuer Protagonisten erlaubte.

Weiterhin verteilte sich die Gruppe der retornados in Portugal entsprechend bestehender familiärer Bindungen. Auf diese Weise konnte für die rückkehrenden Siedler ein Mechanismus familiärer Solidarität wirksam werden; vermutlich auch aufgrund der kurzen Dauer der Emigrationserfahrung in vielen Fällen zeigte sich, dass die Herkunftsfamilien noch immer soziale Solidarität pflegten. Manche Beobachter sprachen gar davon, dass in diesem Fall eine echte „Familienwohlfahrt“ anstelle des Wohlfahrtsstaates zum Tragen komme. ${ }^{42}$ In der Frage der regionalen Verteilung der retornados zeigte sich eine durchaus geschickte politische Planung. Weil eine Konzentration vorübergehend obdachloser Rückkehrer um jeden Preis vermieden werden sollte, wurde eine gleichmäßige Verteilung der retornados innerhalb Portugals in die Wege geleitet. $^{43}$

Die im Hinblick auf die Eingliederung günstige soziale Zusammensetzung der Gruppe erklärt sich aus den Formen der Siedlerrekrutierung in den letzten drei Jahrzehnten des Estado Novo. Der Mechanismus des Einladungsschreibens, der sogenannten carta de chamada, der den Zugang zur Niederlassung in den Kolonien überhaupt erst erlaubte und bis in die 1960er Jahre hinein gültig war, hatte das Feld der Rekrutierung für diese Territorien definiert und in sozialer wie regionaler Hinsicht die Wanderungsströme gesteuert. Mit der Intensivierung der Siedlermigration in den 1950er und 1960er Jahren hatte die Festlegung auf junge und gut ausgebildete Individuen sich noch verstärkt. Die Tatsache, dass Portugals Staatsbürgerschaftskonzept im Hinblick auf die retornados auf dem ius sanguinis beharrte, favorisierte zudem die Rückkehr solcher Personen, welche in Portugal Familienbande hatten, und damit nach 1975 derartige Bindungen auch zur Abfederung der eigenen sozialen Lage aktivieren konnten. ${ }^{44}$

41 Cláudia Orvalho Castelo, Passagens para África. O povoamento de Angola e Moçambique com naturais da metrópole, Porto 2007.

42 Boaventura Sousa Santos, zitiert nach Miguel Brilhante, As representações sociais do repatriado, Lissabon 1993, S. 86.

43 Ribeiro, Vertigem da descolonização, S. 415.

44 Die Verwendung des ius sanguinis - und damit eines Systems, in welchem die Staatsbürgerschaft auf die Abkömmlinge von Mitgliedern einer Landesbevölkerung beschränkt ist und dem System des ius solis gegenübersteht, welches die Staatsbürgerschaft für alle in einem Land geborenen Kinder eröffnet - wurde durch das Gesetzesdekret [Decreto-lei] 308 - A/75 vom 24.6.1975 in Bezug auf die Rückkehr 
Die Verfügbarkeit anderer Zielgebiete zur Auswanderung für portugiesische Siedler aus Angola und Mosambik hatte ebenfalls einen Effekt. 30.000 portugiesische Bewohner der Kolonien zogen die Flucht nach Südafrika der Migration nach Portugal vor, und etwa weitere 17.000 wählten Brasilien, während kleinere Gruppierungen andere Auswanderungsziele anstrebten. Es ist davon auszugehen, dass diese letzteren Personengruppen unter anderem solche Siedler umfassten, die die größten Schwierigkeiten in der Eingliederung in die Gesellschaft der Metropole erwarten ließen.

Schließlich ist darauf hinzuweisen, dass die Politiken der portugiesischen Regierungen, welche letztlich vor allem Übergangsregierungen waren und sich an klar definierte Personengruppen richteten, darauf zielten, die Entstehungsbedingungen für eine kollektive Aushandlung der Integration in jedem Fall zu verhindern. Das Modell der individualisierten Integration sollte vermeiden, dass die französische Erfahrung einer Bildung von regelrechten Milieus der piedsnoirs sich in Portugal wiederholte. In diesem Zusammenhang findet sich eine Reihe sehr konkreter Maßnahmen. So wurde ein Quadro Geral de Adidos, eine Klasse angegliederter Funktionäre geschaffen, welche der Eingliederung derjenigen retornados diente, die in den Kolonien im öffentlichen Sektor gearbeitet hatten. Andere Mittel umfassten die Vergabe von Spezialkrediten zur Investition und die Einrichtung des Instituts zur Hilfeleistung bei Rückkehr von Staatsbürgern - des Instituto de Apoio ao Retorno de Nacionais (IARN). Die staatliche Politik der Reintegration nahm erhebliche öffentliche Ausgaben in Kauf, die einer Minderheit der rückkehrenden Siedler eine besonders rasche und unproblematische Eingliederung in die Gesellschaft ermöglichten. ${ }^{45}$

In der Breitenwirkung implizierte die Rückkehrwelle im Hinblick auf die demographische Entwicklung Portugals einen ausgleichenden Effekt. Sie wirkte dem tendenziellen Bevölkerungsrückgang entgegen, dynamisierte auf längere Sicht die besonders stark entvölkerten Zonen des Mutterlandes und verringerte regionale Asymmetrien in der Wirtschaftsleistung des Landes. Außerdem

von europäischen Siedlern aus den Kolonien verankert. Dieses Dekret eröffnete die Rücksiedlung aus den ehemaligen Kolonien lediglich den Nachkommen von Portugiesen, nicht jedoch europäischen Siedlern anderer Herkunft, was einer generellen Ersetzung des ius solis durch das ius sanguinis in allgemeinen Gesetzestexten gleichkam. Vgl. Pires, Migrações e integração, S. 128.

45 Kennzeichnend sind hier die Polemiken des Jahres 1975, als viele Angestellte des öffentlichen Dienstes der ehemaligen Kolonien, die kein Funktionärsstatut besaßen, ob der befürchteten „Bevorzugung“ der Beamten während der Wiedereingliederung massive Verärgerung zeigten. Vgl. AHU, Arquivo do Ex IARN, 264, João Machado de Almeida, Funcionários do Ultramar. Quais as Atribuições do Quadro dos Adidos [September 1975]. Solche Mobilisierungseffekte gegen die postrevolutionären Regierungen und ihre Maßnahmen, die erhebliche Sprengkraft bargen, verloren allerdings an Bedeutung, als die breiteren sozialen Aktivitäten des IARN und der Regierung zu greifen begannen. 
bediente sie die neuen Bedürfnisse des Arbeitsmarktes, stärkte quantitativ die Größe der Erwerbsbevölkerung, die zugleich im Durchschnitt jünger und höher gebildet wurde. Dies glich über die Jahre die vom Staat kurz nach der Dekolonisation vergebenen individuellen Integrationskredite an die retornados aus. Auf sozialer Ebene waren nicht nur die neuen Bildungsdifferenzen wirkmächtig, auch das Wertsystem der portugiesischen Familien veränderte sich. Schließlich stärkte die Präsenz der retornados auf parlamentarischer Ebene die politische Rechte beziehungsweise unterstützte ihre Neuentstehung nach den Erfahrungen der Revolution im April 1974, während auf der anderen Seite die Rücksiedler sich politisch vor allem in den gemäßigten Parteien der rechten Mitte wiederfanden. Ein lautstarker Protest auf der extremen Rechten kam nicht zum Tragen.

Selbst unter dem Vorzeichen einer vielleicht "gelungenen“, das heißt relativ konfliktarmen, aber doch von Veränderung der portugiesischen Gesellschaft als ganzer geprägten Rückkehrerfahrung ist es vorstellbar, dass diese Thematik zum Gegenstand einer fortdauernden gesellschaftlichen Diskussion hätte werden können. Dies war, wie gesagt, nicht der Fall - und das Schweigen in den Kommunikationsmedien war in diesem Zusammenhang bereits früh, in den 1970er Jahren, ein bezeichnendes Phänomen. ${ }^{46}$ Der journalistische Diskurs hätte, zur gleichen Zeit, simplifizierende Bilder zur Situation des retorno anbieten können; diese Bilder wiederum hätten Meinungen schaffen und beeinflussen können, und, da die Siedler im politischen Spektrum als Anhänger der politischen Rechten eingeschätzt wurden (obwohl diese Positionierung sich schon in der zweiten Hälfte der 1970er Jahre als wenig dogmatisch erweisen sollte), hätten sie zur Zielscheibe einer aggressiven, revolutionär geprägten Presse werden können. Diese Entwicklung blieb jedoch aus.

Vereinzelt kamen in Presseorganen führende Funktionäre und Politiker des frühen postrevolutionären Portugals mit Aussagen zur Situation der Siedler zu Wort. Anstatt Öl ins Feuer zu gießen und die portugiesische Gesellschaft weiter $\mathrm{zu}$ polarisieren, waren diese Aussagen jedoch im Wesentlichen darauf bedacht, die Gemüter im Mutterland zu beruhigen. Dies war etwa der Fall für die Kommentierung der Siedlerflucht aus Mosambik. In Folge des Abkommens von Lusaka zwischen der antikolonialen Guerillabewegung FRELIMO und der portugiesischen Regierung vom 7. September 1974, welches die Unabhängigkeit Mosambiks vorbereitete, verließen die portugiesischen Siedler in Scharen das Territorium; ihre Empörung über den „Verrat“ der Regierung in Lissabon wurde

46 Eine erste analytische Studie der betreffenden Beiträge in den Zeitungen Primeiro de Janeiro aus Porto und Diário de Notícias aus Lissabon und damit der beiden urbanen Zentren mit der größten Konzentration von Rückkehrern zeigte, dass vom 1.5.1974 bis zum 31.12.1975 lediglich 229 Artikel zum Thema veröffentlicht wurden. Dies entspricht für diese beiden besonders einflussreichen Zeitungen einer monatlichen Anzahl von elf Beiträgen. Siehe Isabel dos Santos Lourenço, Retorno da África portuguesa. Imagem na imprensa, 1974-1975, Masterarbeit, Universidade do Porto 2009. 
jedoch in der nationalen Presse als „vorübergehender emotionaler Zustand“ beschrieben, der sich normalisieren würde und das portugiesische Mutterland kaum betreffen sollte. ${ }^{47}$ So wurde in den Medien der retorno als ein Problem dargestellt, welches bei aller Dramatik sich auf dem Wege zu einer Lösung befand. Rosa Coutinho, portugiesischer Hochkommissar in Angola, kommentierte gar optimistisch im Hinblick auf den Exodus aus diesem Territorium: „[D]ie Dekolonisation von Angola wird ein Beispiel für das südliche Afrika geben. ${ }^{\text {“48 }}$ Von Seiten der Politiker und Funktionäre der unmittelbaren Nachrevolutionsperiode war ganz offensichtlich die Devise ausgegeben worden, die Abreise der Siedler aus Afrika positiv darzustellen und ihre Ankunft in Europa sowie die Probleme ihrer Integration schlicht auszublenden.

Während diese positiven Parolen in der portugiesischen Presse, welche in den 24 Monaten nach dem 25. April 1974 unter einem erheblichen Anpassungsdruck stand, noch nicht allzu sehr überraschen, ist es doch erstaunlich, dass trotz der Dimensionen des Phänomens eine kritische Diskussion in Pressemedien gänzlich fehlte. Angesichts der Tatsache, dass bis zur langsamen Normalisierung der portugiesischen politischen Szenerie um die Jahreswende 1975/76 die rückkehrenden Siedler von den linksgerichteten Militärs der Junta de Salvação Nacional als potenzielle Gegner der neuen politischen Strukturen gesehen wurden, wäre ein eher feindseliger Diskurs durchaus zu erwarten gewesen. Dieser tauchte jedoch in den Presseorganen nur sehr vereinzelt auf. Stattdessen etablierte sich ein einigermaßen hartnäckiges Schweigen in den führenden Zeitungen des Landes, welches auf der einen Seite zur Integration der retornados beitrug, auf der anderen Seite aber auch die öffentliche Erinnerung an die Rückkehrwelle schwach ausfallen ließ. Die relativ geringe Kommentierung der Rücksiedlung von 1974/75 hielt im Übrigen über die folgenden 35 Jahre an. Einen Effekt auf die öffentliche Debatte über Portugals Dekolonisationsprozess konnte das Kapitel des retorno damit vor dem Hintergrund einer vergleichsweise konfliktarmen Integration, die auch in den Medien keine kritische Diskussion erfuhr, nicht ausüben.

\section{Die umkämpfte Geschichte. Populäre Militärhistoriographie, Entzauberung des portugiesischen Mythos und die Wucht der internationalen Kritik}

Es ist als typisches Phänomen in europäischen Gesellschaften nach dem Zweiten Weltkrieg anzusehen, dass die wissenschaftliche Beschäftigung mit potenziell problematischen nationalen Thematiken oftmals mit erheblicher Verzögerung

47 Vítor Crespo, portugiesischer Hochkommissar in Mosambik, zitiert nach Diário de Notícias, 29.10.1974, S. 13.

48 Rosa Coutinho, zitiert nach Diário de Notícias, 18.12.1974, S. 11. 
einsetzt. Die Analyse von kolonialen Strukturen im Vorfeld der Dekolonisationen und der Dekolonisationsprozesse selbst bildet hier keine Ausnahme, sondern kann vielmehr als eine besonders charakteristisches Beispiel angesehen werden. Die verspätete historische Erschließung der kolonialen Erfahrung trifft grundsätzlich für alle europäischen Kolonialreiche zu. Im portugiesischen Fall ist jedoch die Abwendung der Historiographie von „überseeischer Geschichte“ besonders einschneidend, waren doch Portugals Beitrag sowohl zu den descobrimentos, den Entdeckungen in Afrika und im südlichen und östlichen Asien, als auch die portugiesische Rolle in der sozialen und ökonomischen Aufwertung der afrikanischen Kolonien ein jahrzehntelanges intensives Publikationsthema gewesen. Die Jahre nach 1974 sahen dennoch eine Abwendung portugiesischer Historiker von entsprechenden Themen, zum Teil in der Retrospektive als „Europäisierung“ der portugiesischen Geschichtswissenschaft bezeichnet. Nur kurz, im Rahmen der Gedächtnisfeiern zur Entdeckung des Seeweges auf den indischen Subkontinent um das Jahr 1998 herum, wurde dem Sachgebiet in Form einer Publikationswelle zu Portugals - wohlbemerkt frühneuzeitlichen - Aktivitäten in außereuropäischen Räumen mehr Beachtung zuteil. $^{49}$

Die geringe Aktivität, welche Historiker und Sozialwissenschaftler in der Bearbeitung des Themas zeigten, hat, seit dem Ende der nachrevolutionären Turbulenzen 1976, breiten Raum für eine populärwissenschaftliche Behandlung gelassen. Hier ist herauszustellen, dass, obwohl vielfach von anhaltenden, traumatischen Schäden bei portugiesischen Soldaten geschrieben wurde, die Darstellung und Bewertung der Kriegsereignisse zwischen 1961 und 1974 von Seiten der Kriegsteilnehmer jenseits der Offiziersränge kaum erfolgt ist. ${ }^{50}$ Historische Studien, die mündlichen Berichten dieser Art Raum geben könnten, sind immer noch erst in ihren Anfängen. Demgegenüber wird die Beschreibung weitgehend von ranghohen Militärs mit historiographischen Aspirationen dominiert, was zu einer überaus einseitigen Darstellung von Kriegsverläufen wie von Hintergründen führt. Zumindest ist in den neuesten Publikationen dieser Art die katastrophale militärische Situation in Guinea-Bissau am Vorabend der Nelkenrevolution offengelegt, während für den ebenfalls von Misserfolgen gekennzeichneten Kriegsschauplatz Mosambik die Bewertung der militärischen Kräfteverhältnisse im Moment der Revolution bemerkenswert positiv zu Gunsten der portugiesischen Seite bleibt. Es fehlt in diesen Darstellungen generell eine kritische Analyse der repressiven Rahmenbedingungen, die zum Kriegsausbruch führten und auch vor dem Hintergrund von Reformen zwischen

49 Francisco Bethencourt, Deconstrução da memória imperial. Literatura, arte e historiografia, in: Ribeiro, Fantasmas, S. 69-89, hier S. 78-80.

50 Luís Quintais, As guerras coloniais portuguesas e a invenção da História, Lissabon 2000, S. $126-146$. 
1961 und 1974 nicht vollständig verschwanden. ${ }^{51}$ Diese Art der Populärhistorie ist als ein Typus neuer Tradition zwischen 1976 und $2010 \mathrm{zu}$ sehen.

Eine Öffentlichkeit internationaler Historiker fühlte sich gerade vor dem Hintergrund dieser Monopolisierung der Kriegsanalyse durch portugiesische Militärs und Teilnehmer an den Operationen provoziert und $\mathrm{zu}$ scharfen Reaktionen genötigt. Sie speiste sich zum Teil aus einer Gruppe von Historikern und Journalisten, die bereits während der Kriegsjahre klar Partei zu Gunsten der antikolonialen Guerillabewegungen ergriffen hatten. ${ }^{52}$ Nach der Dekolonisation des portugiesischen Kolonialreiches nahm diese Richtung in der internationalen Geschichtswissenschaft dann die weiterreichende koloniale Vergangenheit Portugals in Afrika in den Blick. Zielscheibe war auf der einen Seite die beständige Behauptung von portugiesischer Seite, die sich über die Revolution hinweg halten sollte, dass Portugals Territorien im subsaharischen Afrika im Prinzip ein império multi-racial (ein multiethnisches Imperium) gewesen seien. In äußerst detaillierter Form konterkarierte vor allem Gerald Bender diesen Mythos und arbeitete die verschiedenen Formen der Diskriminierung heraus, denen die afrikanischen Bevölkerungen in Portugals Kolonien ausgesetzt waren. ${ }^{53}$ Flankiert wurde die entsprechende Einschätzung von anderen Autoren, so etwa von David Birmingham, der die portugiesische Assimilationsdoktrin als Ganzes unter Beschuss nahm. ${ }^{54}$

Die Revision der portugiesischen Kolonialpraktiken ging jedoch noch weiter. In den 1980er und 1990er Jahren wurde der Umgang der portugiesischen Verwaltungsbeamten in den afrikanischen Kolonien mit den lokalen Arbeitskräften zum Gegenstand vertiefter Untersuchungen. Obschon die Analyse nicht allzu materialreich verlief und, zumal im Falle Angolas, das sehr aufschlussreiche und von Kolonialfunktionären produzierte Archivmaterial fast noch unerschlossen ist, wurde die Idee einer besonders empathischen Behandlung

51 Die letzten Erzeugnisse dieser populären Kriegshistoriographie, welche den portugiesischen Buchmarkt einigermaßen dominiert, sind Fernando Policarpo, A guerra de Guiné, 1963-1974, Lissabon 2010 und Francisco Proença Garcia, A guerra de Moçambique 1964-1974, Lissabon 2010. Bei den Autoren der neueren Generation handelt es sich um etablierte Militärwissenschaftler, die dennoch die kritische Analyse sozialer Hintergründe auf afrikanischem Terrain nahezu gänzlich ignorieren.

52 Gérard Chaliand, Lutte armée en Afrique, Paris 1964, S. 33-41; John Marcum, The Angolan Revolution, Bd. 1: The Anatomy of an Explosion, 1950 - 1962, Cambridge, MA 1969; Basil Davidson, In the Eye of the Storm. Angola's People, London 1972, eine deutschsprachige Zusammenfassung von Davidsons Positionen findet sich in ders., Eine Million Portugiesen nach Afrika? Guerilla-Kriege gegen die Herrschaft der Weißen, in: Der Spiegel 46. 1968, S. $127-130$.

53 Gerald J. Bender, Angola Under the Portuguese. The Myth and the Reality [1978], London 2004, S. 199-204.

54 Siehe die Zusammenfassung dieser Positionen in David Birmingham, Portugal e África, Lissabon 2003. 
der afrikanischen Bevölkerungen durch portugiesische Funktionäre entschieden widerlegt. Im Gegenteil standen ausgesprochen brutale Vorgehensweisen, insbesondere im Hinblick auf die Ausbeutung von Zwangsarbeitern, im Zentrum der Analyse: Historiker wie vor allem Allen F. Isaacman haben eine Fülle von Belegen für diese Missbräuche ausfindig gemacht, besonders Mosambik wurde zum geographischen Feld derartiger Fallstudien. ${ }^{55}$ Zwischen dem Ende der 1970er Jahre und der zweiten Hälfte der 1990er Jahre veränderte sich damit die Betrachtung der Kolonialpolitik Portugals von Seiten der internationalen Geschichtswissenschaft und zeigte erste Ansätze, eine im besonderen Maße rückständige, missbräuchliche und brutale Praxis offenzulegen. Angesichts des internationalen Gegenwindes - der in dieser Form für die Diskussion einer kolonialen Erfahrung auf internationaler Ebene völlig singulär ist überrascht es dann auch nicht, dass portugiesische Geschichtswissenschaftler sich aus dem gesamten Diskussionsfeld weitgehend zurückgezogen haben. Diese Entwicklung sorgte jedoch dafür, dass über Jahre und Jahrzehnte hinweg breitere, von portugiesischen Wissenschaftlern ausgehende Analysen sowohl des Dekolonisationsprozesses wie auch des späten portugiesischen Kolonialstaates fehlen.

\section{Schlussfolgerungen}

Die Tatsache, dass in Portugal der Zusammenbruch des Kolonialreiches maßgeblich an eine politische Revolution und den Sturz eines autoritären Regimes in der Metropole gekoppelt war, gibt dem portugiesischen Fall eine grundverschiedene Ausrichtung im Vergleich zu allen anderen europäischen Dekolonisationsprozessen. Die Vehemenz, mit welcher die beiden Premierminister António Oliveira Salazar und Marcello Caetano in ihrer Rhetorik das Wohl und Wehe des Estado Novo stets mit dem Machterhalt über Portugals afrikanischen Kolonialbesitz verbunden hatten, hätte dazu führen können, dass mit dem Sturz der Diktatur auch die Praktiken in der Beherrschung von Kolonialbevölkerungen angegriffen worden wären. Zumal Dekolonisation und Aufgabe der Siedlungsprojekte im subsaharischen Afrika nicht das völlige Ende portugiesischer Initiativen - staatlicher wie privater - in den nun unabhängigen Staaten des „lusophonen“ Afrikas bedeuteten. Eine solche Aufarbeitung wurde jedoch in der portugiesischen Bevölkerung wie unter den sozialen Eliten jeglicher politischer Couleur nach dem Ende der Revolutionsperiode 1976 nicht

55 Eine Auswahl dieser kritischen Literatur bilden etwa Allen F. Isaacman, Chiefs, Rural Differenciation and Peasant Protest. The Mozambican Forced Cotton Regime, 1938-1961, in: African Economic History 14. 1985, S. 15-56; Allen F. Isaacman u. Barbara Isaacman, Mozambique. From Colonialism to Revolution, 1900 - 1982, Boulder, CO 1983; sowie Leroy Vail und Landeg White, “Tawani, Machambero!" Forced Cotton and Rice Growing on the Zambezi, in: Journal of African History 19. 1978, S. 239-263. 
geleistet. Stattdessen sind die folgenden Dekaden von einem Rückzug der populären wie der akademischen Diskussionen von der Dekolonisationsphase und von der Erfahrung des späten portugiesischen Kolonialstaates geprägt. Der Trend verläuft erst langsam und unter anderem angesichts des fünfzigjährigen Jubiläums des Ausbruchs der ersten antikolonialen Aufstände in Angola zum Beginn der Dekolonisationskriege wieder in die Gegenrichtung.

Einige Besonderheiten des portugiesischen Kolonialreiches wie des nachrevolutionären politischen und sozialen Prozesses sind dazu geeignet, diese blockierte Erinnerung zu erklären. Zunächst war die koloniale Realität von massiven Widersprüchen geprägt: ein spät einsetzender Reformprozess stand dem Ziel des Salazar-Regimes gegenüber, Angola und Mosambik zu Siedlerkolonien zu machen. Die Kolonialadministration vollzog zwischen 1945 und 1974 mehrere Wenden in ihrer grundsätzlichen und mehrheitlichen Ausrichtung, verfolgte aber lediglich unter dem Druck der Militärs, die anhaltende soziale Unzufriedenheiten fürchteten, einen wirklich reformistischen Kurs. Zwangsarbeit und Körperstrafen unter dem Indigenat waren weit verbreitete Praktiken innerhalb des Kolonialreiches, die erst 1961/62 abgeschafft wurden und noch längere Zeit im lokalen Gedächtnis blieben. Dies machte es nach der Revolution grundlegend schwierig, sich mit den Charakteristiken des Kolonialreiches in einer gesellschaftlichen Debatte auseinanderzusetzen, hätte man doch gegenüber Hunderttausenden vom Krieg betroffenen Familien zugeben müssen, dass man für ein repressives Kolonialregime gekämpft hatte. Die Deutungsangebote ehemaliger hoher kolonialer Würdenträger, die sich nach ihrer Rehabilitierung betont technisch mit der kolonialen Erfahrung auseinandersetzten und sie, wie Adriano Moreira, mit paternalistischen Perspektiven etwa gegenüber der Bevölkerung der unabhängigen Republik der Kapverdischen Inseln verbinden konnten, waren demgegenüber beruhigend und attraktiv. Entsprechende Positionen halten vor diesem Hintergrund bis zum heutigen Tag einen wichtigen Platz in öffentlichen Sichtweisen auf Portugals Ultramar.

Die umwälzende Erfahrung des retorno hätte dennoch eine breitere gesellschaftliche Debatte notwendig machen können, stellten die Rücksiedler doch eine zahlenmäßig gewaltige Gruppe dar, die zudem in einen ökonomisch ungünstigen Kontext trat. Die Spezifika der portugiesischen Siedlungspolitik verhinderten jedoch Ausbrüche sozialen Unfriedens im Rahmen des Rückkehrprojektes. Rücksiedler, die aufgrund ihres relativ hohen Bildungsstandes und ihres sozialen Gesamtprofils für einen sich wandelnden Arbeitsmarkt letztlich wie gerufen kamen, konnten, zudem günstig verteilt, vergleichsweise rasch in die portugiesische Gesellschaft integriert werden. Die Tatsache, dass die postrevolutionären Medien sich in der Bewertung des Phänomens stark zurückhielten - und dies, obwohl die retornados von vielen Mitgliedern der Junta de Salvação Nacional und linksorientierten Politikern als potenziell gefährlich und als künftige Anhänger der politischen Gegenseite betrachtet wurden - war vermutlich mitentscheidend für die von sozialen Unruhen freie Einbindung der Rücksiedler. 
Im akademischen Bereich wurde die portugiesische Auseinandersetzung mit der Thematik offensichtlich durch die Konfrontation mit einer internationalen detaillierten, kritischen Würdigung der repressiven portugiesischen Praktiken eher geschwächt als gestärkt. Portugiesische Wissenschaftler zogen sich in der Folge aus dem Bereich der Kolonialgeschichtsschreibung zurück. Damit wurde das portugiesische Feld den Autoren populärgeschichtlicher Werke überlassen und auf die kritisch-empirische Analyse praktisch vollständig verzichtet. Durch diese Form der vor allem von ehemaligen Militärs dominierten „nationalen“ Aufarbeitung wurde die Blockade in der portugiesischen Kolonialerinnerung weiter verstetigt. Obwohl die in den letzten Jahren $\mathrm{zu}$ erkennende langsame Wiederannäherung an die Thematik eine Veränderung im Umgang mit dieser für das nächste Jahrzehnt erwarten lässt, ist eine solche Entwicklung keineswegs ausgemacht. Als hilfreich erweisen könnte sich in diesem Zusammenhang ein verstärktes Auftreten afrikanischer intellektueller Eliten in der Debatte. Bislang existiert zwischen der Geschichtswissenschaft und ihren Nachbardisziplinen sowie in den Erinnerungsdebatten in den ehemaligen afrikanischen Kolonialterritorien einerseits und der portugiesischen Beschäftigung mit der Dekolonisationsphase und dem Kolonialstaat während der Diktatur des Estado Novo andererseits praktisch keinerlei Verbindung. Die historischen Diskurse im großen, ökonomisch bedeutenden Flächenstaat Angola oder im von kulturpolitischen Initiativen ohnehin recht stark geprägten Mosambik könnten die portugiesische Geschichtswissenschaft und längerfristig auch die portugiesische Öffentlichkeit zu einem verstärkten Engagement in der Aufarbeitung von Kolonialgeschichte zwingen. Diese Neuausrichtung wäre dann eine Folge neuer Realitäten, welche man der Globalisierung zuschreiben kann.

Isabel dos Santos Lourenço, M. A., CEAUP, Faculdade de Letras da Universidade do Porto, P-4150-564 Porto

E-Mail: mslisabel@gmail.com

PD Dr. Alexander Keese, CEAUP, Faculdade de Letras da Universidade do Porto, P-4150-564 Porto

E-Mail: a.keese@gmx.eu 